\title{
Usefulness of contrast-enhanced ultrasonography in the diagnosis of ruptured hepatocellular carcinoma
}

\author{
Kazue Shiozawa $\cdot$ Manabu Watanabe $\cdot$ \\ Takashi Ikehara $\cdot$ Yasushi Matsukiyo $\cdot$ \\ Koji Ishii · Yoshinori Igarashi · Yasukiyo Sumino
}

Received: 1 April 2013/Accepted: 2 June 2013/Published online: 25 June 2013

(C) The Author(s) 2013. This article is published with open access at Springerlink.com

\begin{abstract}
The identification of bleeding sites of ruptured hepatocellular carcinoma (HCC) is important for immediate treatment. We experienced a case of ruptured HCC readily treated with transarterial embolization (TAE) after identification of the bleeding site using contrast-enhanced ultrasonography (CEUS) with Sonazoid. We report the case of a 61-year-old male with multiple HCCs caused by alcohol-related cirrhosis, who was admitted for rapid development of abdominal fullness. The diagnosis was established by hemorrhagic ascites by abdominal paracentesis. No clear extravasation was found on contrastenhanced computed tomography. CEUS using the reinjection method in the post-vascular phase showed active bleeding from a lesion close to the S5 gallbladder bed. Abdominal angiography was urgently performed. Based on CEUS findings, selective cannulation of the cystic artery was performed. Cystic angiography findings with carbon dioxide showed extravasation. He was treated by TAE. Our case had multiple HCCs, but CEUS was useful for the identification of bleeding sites.
\end{abstract}

Keywords Hepatocellular carcinoma - Rupture . Contrast-enhanced ultrasonography · Sonazoid . Transarterial embolization

K. Shiozawa $\cdot$ M. Watanabe $(\bowtie) \cdot$ T. Ikehara · Y. Matsukiyo · K. Ishii · Y. Igarashi · Y. Sumino Division of Gastroenterology and Hepatology, Department of Internal Medicine, Toho University Medical Center, Omori Hospital 6-11-1, Omorinishi, Ota-ku, Tokyo 143-8541, Japan e-mail: manabu62@med.toho-u.ac.jp

\section{Introduction}

Ruptured hepatocellular carcinoma (HCC) requires immediate diagnosis and treatment due to its high mortality. The diagnosis is comparatively easy when made based on clinical course, symptoms, and demonstration of hemorrhagic ascites by abdominal paracentesis, but identification of sites of bleeding is important for immediate treatment. Contrast-enhanced computed tomography (CECT) is performed for identification of these sites in many cases because a wide area can be observed [1]. However, CECT is invasive because of iodine allergy, exposure to radiation, and decreased renal function by administration of the contrast agent.

Contrast-enhanced ultrasonography (CEUS) is a noninvasive alternative approach that may be useful for identification of bleeding sites [2,3]. We experienced a case of ruptured HCC that developed as a complication of alcoholrelated cirrhosis. The patient had multiple HCCs, but the culprit artery was readily treated with transarterial embolization (TAE) after identification of the bleeding site using CEUS with Sonazoid (Daiichi Sankyo Pharmaceutical, Tokyo, Japan). Here, we describe the case with a literaturebased discussion.

\section{Case report}

The patient was a 61-year-old male. He had no relevant medical or family history, but he was a heavy drinker who consumed $>2 \mathrm{~L}$ of beer every day. He visited a nearby hospital for a chief complaint of general malaise and was diagnosed with multiple HCCs caused by alcohol-related cirrhosis. Approximately 1 month after the first visit, he was admitted to our hospital for rapid development of 
abdominal fullness. Physical findings at admission showed blood pressure 148/98 $\mathrm{mmHg}$, pulse rate $64 \mathrm{bpm}$, ascites retention, and a palpable fist-sized tumor in the epigastric region. Blood tests upon admission showed $\mathrm{Hb} 10.4 \mathrm{~g} / \mathrm{dl}$ (normal 14.0-17.0 g/dl), AST $55 \mathrm{U} / 1$ (normal 12-35 U/l), ALT $35 \mathrm{U} / 1$ (normal 6-31 U/l), $\gamma-\mathrm{GTP} 283 \mathrm{U} / 1$ (normal 9-58 U/l), T-Bil $4.9 \mathrm{mg} / \mathrm{dl}$ (normal $0.1-1.0 \mathrm{mg} / \mathrm{dl}$ ) and PT $\% 68 \%$ (normal $\geq 70 \%$ ), suggesting mild anemia and increased hepatobiliary enzymes. Hepatic function was evaluated as C (10 points) in the Child-Pugh classification. Tumor markers were high, as shown by the levels of AFP and PIVKA II of $27.4 \mathrm{ng} / \mathrm{ml}$ (normal $0.0-15.0 \mathrm{ng} / \mathrm{ml}$ ) and $567 \mathrm{mAU} / \mathrm{ml}$ (normal $<40 \mathrm{mAU} / \mathrm{ml}$ ), respectively. HBV and $\mathrm{HCV}$ were negative and renal function was normal. Hemorrhagic ascites was found in abdominal paracentesis and the case was diagnosed as ruptured HCC.

Abdominal CECT showed a large HCC with a maximum diameter of $15 \mathrm{~cm}$ in S7/8 of the liver and multiple HCCs in both lobes (Fig. 1). The attenuation value of the ascites was as high as 30 Hounsfield units, but no clear extravasation was found in the arterial and equilibrium phase, and thus the bleeding site could not be identified. Abdominal ultrasonography also showed multiple tumors in both lobes and ascites with high echo density (Fig. 2a). However, even with these data the bleeding site was unclear, and CEUS was performed for the large HCC in S7/ 8 as the region of interest. The ultrasound equipment used in this examination was SSA-790A (Toshiba Medical Systems, Tokyo, Japan) with a convex probe (PVT-375BT, 3.75-MHz center frequency). The imaging mode used was wideband harmonic imaging (pulse subtraction) with transmission/reception frequencies of 1.8 and $3.5 \mathrm{MHz}$, respectively. The mechanical index for acoustic output was set to 0.2 ; the dynamic range was set to $60-65 \mathrm{~dB}$. A intravenous bolus injection of Sonazoid $(0.5 \mathrm{ml})$ was administered via a left cubital venous line followed by $10 \mathrm{ml}$ normal saline flush. Observation in the arterial phase showed no clear leakage of contrast agent from this tumor,
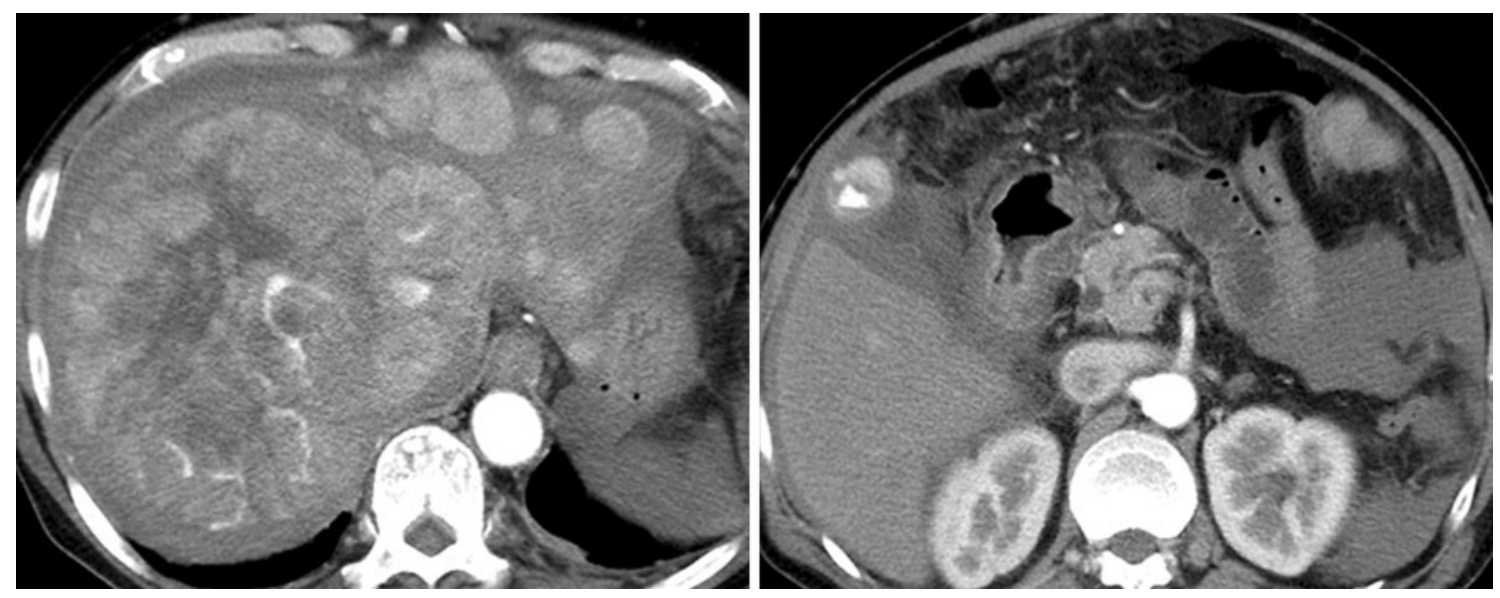

Fig. 1 Dynamic computed tomography scan in the arterial phase showed multiple hypervascular lesions in both hepatic lobes with highly attenuated peritoneal fluid

Fig. 2 a Gray-scale ultrasonography demonstrated a lesion closed to the S5 gallbladder bed (arrow heads) with fluid. b Contrast-enhanced ultrasonography (CEUS) using the re-injection method with Sonazoid in the post-vascular phase showed pulsating active bleeding as extravasation of microbubbles (arrow) from this lesion (arrow heads) into the abdominal cavity
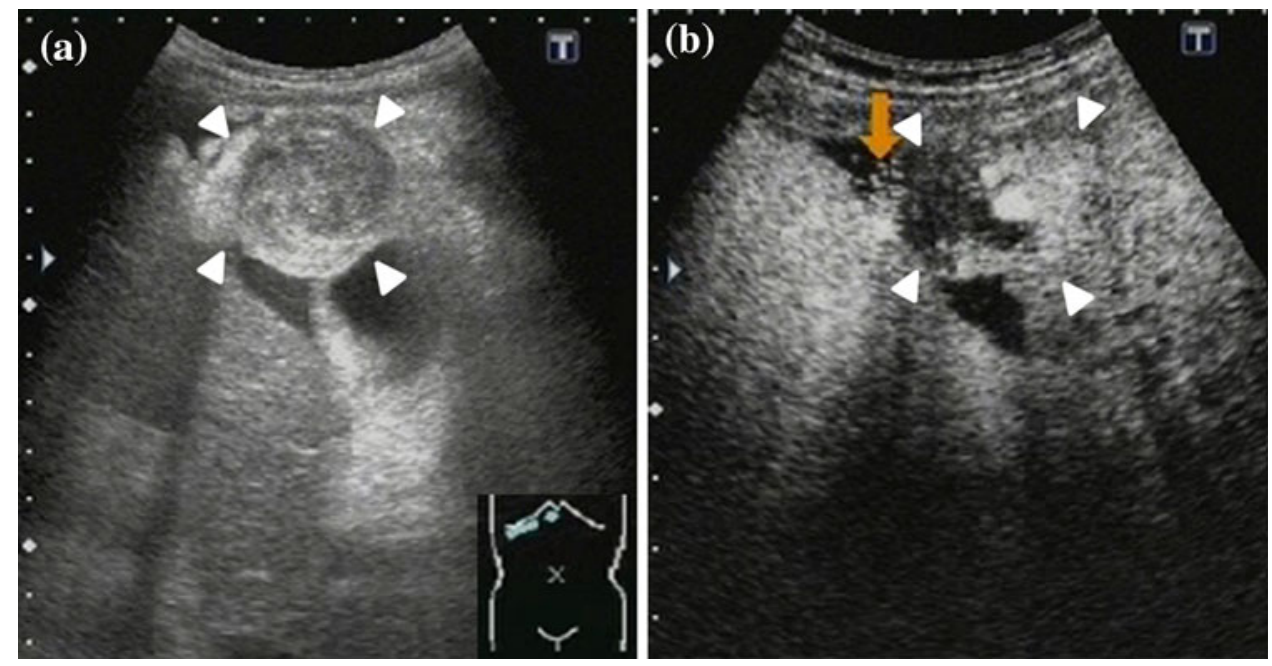
Fig. 3 a Cystic angiography findings showed multiple tumor stains without extravasation of contrast agent. b Cystic angiography findings with carbon dioxide showed extravasation (arrow). These findings agreed with the CEUS findings
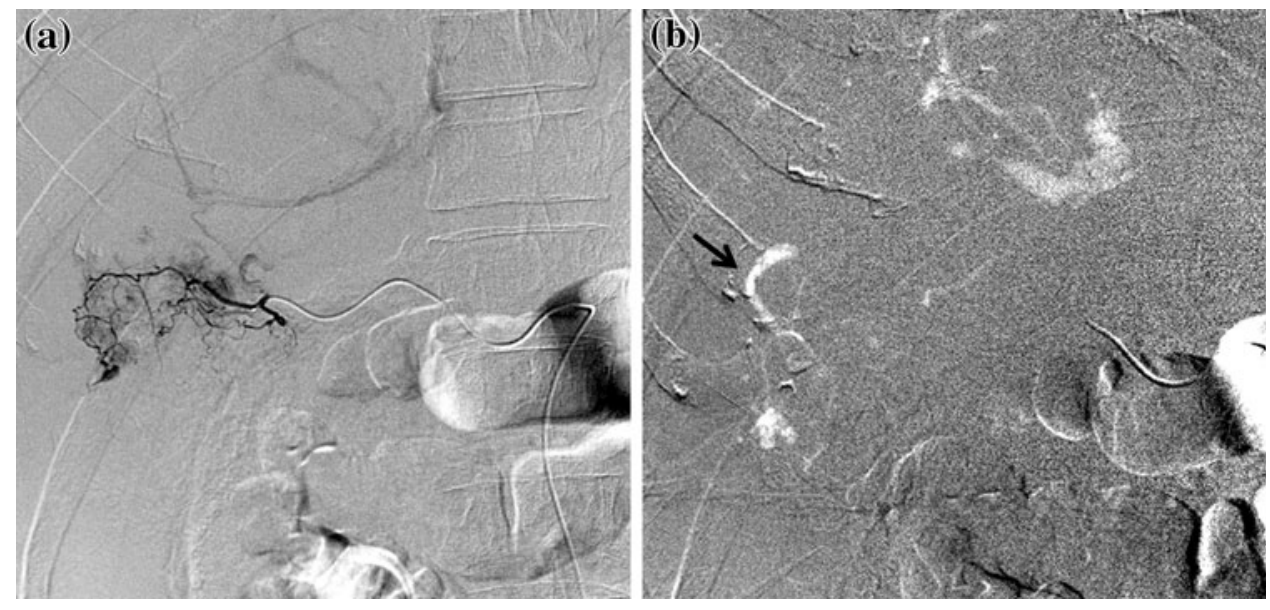

but punctate high-intensity echoes of Sonazoid microbubbles were observed in ascites. In particular, extremely highintensity echoes of Sonazoid microbubbles were present in ascites surrounding the tumor, close to the S5 gallbladder bed. The tumor was imaged again using the re-injection method [4] with Sonazoid in the post-vascular phase and leakage of the contrast agent from the tumor into the abdominal cavity was observed, indicating that this was the site of bleeding (Fig. 2b).

Abdominal angiography was urgently performed. Imaging of the celiac artery and hepatic artery showed no clear extravasation. However, since the tumor on the gallbladder bed was suspected as the bleeding site based on CEUS, selective cannulation of the cystic artery was performed (Fig. 3a). The artery was imaged using carbon dioxide $\left(\mathrm{CO}_{2}\right)$, which is a highly sensitive method for evaluation of blood flow [5]. Leakage of $\mathrm{CO}_{2}$ out of the vessel was apparent, confirming that the bleeding site was the tumor in S5, which was fed by the cystic artery (Fig. 3b). TAE was conducted using a gelatin sponge (Gelpart; Astellas Pharma, Tokyo, Japan) with a particle size of $1 \mathrm{~mm}$, and the clinical course after TAE was favorable with arrest of bleeding.

\section{Discussion}

Ruptured HCC is a common cause of acute non-traumatic intra-abdominal hemorrhage and has an incidence of about $10 \%$ in patients with terminal HCC [6]. Ruptured HCC causes severe intraperitoneal bleeding and acute hepatic failure with a poor prognosis, and therefore immediate diagnosis and treatment are required. Symptoms include sudden epigastric pain or right hypochondrial pain caused by rapid expansion of Glisson's capsule. Hemorrhagic shock is observed in 59-90\% of patients with ruptured HCC, peritoneal irritation and abdominal prominence occur in 60-100\%, and hemorrhagic ascites is present in almost all patients [7, 8]. Ruptured HCC should be the first suspected condition when such symptoms occur in patients with cirrhosis or HCC.

Ruptured HCC may be a rupture of a parasitic artery or vein, such as the inferior phrenic vessel, feeding or draining the tumor, with accompanying hemorrhage [9]. This rupture may be caused by secondary trauma to a tumor on the surface of the liver or increased pressure within a tumor due to rapid tumor growth causing invasion and obstruction of a hepatic vein [10]. The risk of rupture is particularly high in patients with large HCC on the hepatic surface, and the clinical course of such cases should be observed carefully, taking this risk into account. In our case with multiple HCCs, ruptured HCC was suspected and abdominal paracentesis was performed because of rapid onset of abdominal fullness, which suggested hemorrhagic ascites, despite no abdominal pain or decreased blood pressure.

Identification of the bleeding site is important to facilitate immediate treatment in a case of suspected ruptured HCC. This is commonly achieved with CECT [11], but there is a possibility that a bleeding site may not be apparent in an image due to the imaging timing, even if active hemorrhage is present. This is because CECT has superior spatial resolution, but requires a certain period of time for imaging. Several recent reports have suggested that CEUS with Levovist (Schering, Berlin, Germany) or Sonazoid may also be useful for this purpose [2, 3]. In 10 patients with ruptured HCC who underwent CEUS with Levovist and abdominal angiography, Matsumoto et al. [2] found that CEUS could be used to differentiate active hemorrhage from inactive hemorrhage by examining the contrast agent in ascites. Sugihara et al. [3] found that CEUS with Sonazoid was effective for identification of the bleeding site in a hepatic surface after radiofrequency ablation (RFA) based on a jet-like high-intensity echo in pleural effusion, although the findings could not be confirmed with color Doppler imaging. Other reports [12] have suggested the usefulness of CEUS with Levovist for large 
ruptured $\mathrm{HCC}$ in cases of single tumors, large tumors, or tumors in which bleeding sites were identified for bleeding caused by RFA.

The high temporal resolution of CEUS is useful for evaluation of hemodynamics, but identification of bleeding sites is difficult in patients with multiple HCCs because CEUS evaluates only a single cross-sectional image. However, the bleeding site in our case could not be identified with CECT because of the presence of multiple HCCs, in contrast to previous cases, whereas CEUS enabled identification of the bleeding site. In our case, active hemorrhage was suspected because of leakage of Sonazoid bubbles in ascites observed in the arterial phase. This led to a detailed examination of potential HCC under the hepatic surface, which has a risk of rupture in a case with multiple HCCs. Many Sonazoid bubbles were found in ascites around HCC on the S5 gallbladder bed, and thus bleeding from this site was suspected. Sonazoid was again intravenously injected using the re-injection method with this site as an area of interest and leakage of contrast agent from the tumor surface was confirmed. In subsequent abdominal angiography, selective cannulation was performed for the cystic artery based on CEUS findings and imaging with $\mathrm{CO}_{2}$ showed extravasation from the HCC. Immediate arrest of bleeding was achieved with TAE.

Identification of bleeding sites is difficult in many cases. Extravasation may not be observed on CECT in patients with multiple HCCs, as seen in our case, but bleeding sites may be indicated by Sonazoid microbubbles in the ascites. The re-injection method [4] with Sonazoid to examine the potential bleeding site in the post-vascular phase in CEUS permits the site to be identified rapidly, even in patients with multiple HCCs.

In conclusion, we experienced a case of ruptured HCC. The patient had multiple HCCs, but the culprit artery was readily treated with TAE after identification of the bleeding site using CEUS with Sonazoid.

Conflict of interest The authors declare that they have no conflict of interest.
Open Access This article is distributed under the terms of the Creative Commons Attribution License which permits any use, distribution, and reproduction in any medium, provided the original author(s) and the source are credited.

\section{References}

1. Casillas VJ, Amendola MA, Gascue A, Pinnar N, Levi JU, Perez JM. Imaging of nontraumatic hemorrhagic hepatic lesions. Radiographics. 2000;20:367-78.

2. Matsumoto N, Ogawa M, Nakagawara H, Hiroi Y, Yamamoto T, Arakawa Y, et al. Clinical efficacy of contrast-enhanced ultrasonography (CEUS) in the diagnosis of ruptured hepatocellular carcinoma (HCC). J Med Ultrasonics. 2007;34:101-5. doi: 10.1007/s10396-007-0136-0.

3. Sugihara T, Koda M, Tokunaga S, Matono T, Nagahara T, Ueki $\mathrm{M}$, et al. Contrast-enhanced ultrasonography revealed active thoracic bleeding. J Med Ultrasonics. 2010;37:143-5. doi: 10.1007/s10396-010-0257-8.

4. Kudo M. Hepatocellular carcinoma 2009 and beyond: from the surveillance to molecular targeted therapy. Oncology. 2008;75(Suppl 1):1-12. doi:10.1159/000181865.

5. Terayama N, Matsui O, Ueda F, Hattori Y, Nishijima H, Sanada J. CO2 demonstration of multiple extravasations into a subcapsular hematoma of the liver. Cardiovasc Intervent Radiol. 2004;27:278-81.

6. Zhu LX, Wang GS, Fan ST. Spontaneous rupture of hepatocellular carcinoma. Br J Surg. 1996;83:602-7 (PMID:8689200).

7. Dewar GA, Griffin SM, Ku KW, Lau WY, Li AK. Management of bleeding liver tumours in Hong Kong. Br J Surg. 1991; 78:463-6.

8. Miyamoto M, Sudo T, Kuyama T. Spontaneous rupture of hepatocellular carcinoma: a review of 172 Japanese cases. Am J Gastroenterol. 1991;86:67-71.

9. Kanematsu M, Imaeda T, Yamawaki Y, Seki M, Goto H, Sone Y, et al. Rupture of hepatocellular carcinoma: predictive value of CT findings. AJR Am J Roentgenol. 1992;158:1247-50.

10. Chearanai O, Plengvant U, Asavanich C, Damrongsak D, Sindhvananda K, Boonyapisit S. Spontaneous rupture of primary hepatoma: report of 63 cases with particular reference to the pathogenesis and rationale treatment by hepatic artery ligation. Cancer. 1983;51:1532-6.

11. Kim HC, Yang DM, Park SJ. The various manifestations of ruptured hepatocellular carcinoma: CT imaging findings. Abdom Imaging. 2008;33:633-42. doi:(10.1007/s00261-007-9353-7).

12. Skarin A. Diagnosis in oncology. J Clin Oncol. 2002;19:4108-14. 